\title{
Omalizumab treats chronic rhinosinusitis with nasal polyps and asthma together-a real life study*
}

\author{
Therese Bidder', Jagdeep Sahota1,3, Catherine Rennie², Valerie J. Lund ${ }^{2,3}$, \\ Douglas S. Robinson', Harsha H. Kariyawasam ${ }^{1,2,3}$ \\ Rhinology 56: 42-45, 2018 \\ https://doi.org/10.4193/Rhino17.139 \\ 1 Thoracic Medicine, University College London Hospital NHS Foundation Trust, London, United Kingdom \\ *Received for publication: \\ Rhinology Section, Royal National Throat Nose and Ear Hospital London, London, United Kingdom \\ June 27, 2017 \\ ${ }^{3}$ University College London, London, United Kingdom \\ Accepted: November 16, 2017
}

\begin{abstract}
Introduction: Chronic rhinosinusitis with nasal polyps (CRSwNP) and asthma often coexist and thus treating both with one intervention is an attractive strategy.
\end{abstract}

Objective: To prospectively evaluate whether treatment with the monoclonal antibody against IgE Omalizumab for severe allergic asthma also effectively treats co-existent CRSwNP.

Methods: SNOT-22 and the ACQ-7 scores were recorded at 4 and 16 weeks of treatment in a cohort of patients with both CRSwNP and severe refractory allergic asthma treated with Omalizumab $(n=13)$ according to UK guidelines for their severe asthma. SNOT22 in a surgery only treated CRSwNP with asthma group $(n=24)$ was compared.

Results: Rapid improvement was seen at 4 weeks and 16 weeks of treatment in both CRSwNP and asthma control. The improvement in CRSwNP with Omalizumab was similar to that seen in a group of patients who received upper airway surgery.

Conclusion: Omalizumab treatment for severe allergic asthma also improves co-existent CRSwNP. Further clinical studies of current and emerging biological agents for severe asthma should include upper airway outcomes. These agents may be effective for severe CRSWNP and comparative studies with surgery are warranted.

Key words: Omalizumab, monoclonal antibody, nasal polyps, asthma, outcomes

\section{Introduction}

Chronic rhinosinusitis with nasal polyps (CRSwNP) is difficult to treat, and significantly impacts on patients' lives. Many patients require frequent systemic steroids and recurrent surgery ${ }^{(1)}$. Thus CRSwNP represents a significant clinical and economic burden and there is an urgent need for disease intervention that is both rapid and sustained in its action. CRSwNP is frequently associated with asthma, and often this asthma phenotype is severe ${ }^{(2)}$. Given the mucosal similarities and likely overlapping immune dysregulation that occurs in the upper and lower airway together, termed 'the one airway' concept, it is possible that biologics which target key immunological players in the inflammatory cascade can modulate disease in several tissue settings. If true, one drug has the potential to treat both the upper and lower airway together and thus be cost-effective in the long term. Omalizumab binds IgE and prevents it from binding the $\lg \mathrm{E}$ receptor and thus inhibits the allergic cascade.

Using the sino-nasal outcome (SNOT)-22 and asthma control questionnaire (ACQ)-7, we report here our real life experience of using Omalizumab in our population of severe CRSwNP with severe treatment refractory allergic asthma. We compare the improvement to surgical intervention.

\section{Materials and methods}

All patients with both CRSwNP and severe allergic asthma who underwent Omalizumab therapy were evaluated in a prospective manner. CRSwNP diagnosis was with endoscopic confirmation. Severe asthma was confirmed based on criteria set in the 
ERS-ATS guidelines ${ }^{(3)}$. In order to fulfil the prescribing criteria of Omalizumab for severe refractory allergic asthma, all patients must be sensitised to at least one perennial aeroallergen (for example cat or dust mite). Omalizumab was administered using standard dosing protocols using total serum IgE and weight in kilograms to determine frequency (monthly or 2 weekly subcutaneous injections) and dose of the drug. Thus the Omalizumab dose that would most likely lead to a treatment response in the patient was administered. The lgE range had to be equal to or within 30 to $1500 \mathrm{IU} / \mathrm{ml}$ within a specific weight group to match. SNOT-22 scores at baseline, 4 weeks and 16 weeks of treatment were documented. Patients with CRSwNP who underwent functional endoscopic sinus surgery with polypectomy, like the Omalizumab treated group, were also evaluated with the SNOT-22 score before and after surgery (at the routine surgical follow-up clinic between 4 and 16 weeks). A total SNOT-22 score of over 50 was defined as severe CRSwNP ${ }^{(4)}$. Asthma control was assessed via the ACQ-7 where a mean score of 1.5 and above represents inadequately controlled disease and a score of 0.75 indicates good control.

\section{Statistical analysis}

SNOT-22 is expressed as the median (range). The Mann-Whitney $\mathrm{U}$ test was used to compare non-paired data. All paired withinsubject data was analysed using the Wilcoxon signed rank test. Analysis was with GraphPad Prism version 4 (GraphPad Software Inc., San Diego, CA). Significance was accepted as $\mathrm{p}<0.05$.

\section{Results}

All patients had CRSWNP. None had eosinophilic granulomatous polyangiitis (Churg Strauss Syndrome). Patients with obvious fungal driven disease were excluded. Table 1 summarises the phenotypic characteristics of the two treatment groups. CRSwNP was severe in both treatment groups. The median SNOT-22 at baseline was 52.00 (range 27-78) in the Omalizumab group and 70 (range 26-104) in the surgery only group $(p=0.02)$ (Figure1 A).

Omalizumab leads to a rapid improvement in the SNOT-22 in patients with CRSwNP. This improvement is seen early post Omalizumab and sustained with continued treatment (Figure1 B). The median SNOT-22 decreased to 24.50 (range 1-42) at 4 weeks and 30 (range $3-60)$ at 16 weeks of treatment $(p=0.0033$ and $\mathrm{p}=0.0086$ respectively).

The median SNOT-22 was 29.50 (range 1-61) $(p<0.001$ ) (Figure1 C) post-surgery. There was no difference in the median SNOT-22 between the Omalizumab and surgery treated groups (Figure1 D).

Rapid asthma improvement occurs alongside as reflected in the significantly decreased ACQ-7 at both 4 weeks and 16 weeks of treatment ( $p=0.005$ and $p=0.03$ ), respectively (Figure $1 E$ ).
Table 1. Summary of treatment group CRSwNP phenotypes.

\begin{tabular}{|lcc|}
\hline \multicolumn{1}{|c}{ Phenotype Features } & Omalizumab & Surgery \\
\hline Age (mean years) & 46 & 47.8 \\
\hline Number (sex) & $\begin{array}{c}13 \text { ( } \mathrm{n}=5 \text { male } \\
\mathrm{n}=8 \mathrm{female})\end{array}$ & $\begin{array}{c}24(\mathrm{n}=14 \text { male } \\
\mathrm{n}=10 \mathrm{female})\end{array}$ \\
\hline Total serum IgE (median) & $396.5 \mathrm{IU} / \mathrm{L}$ & $268.0 \mathrm{IU} / \mathrm{L}$ \\
\hline Eosinophil count (median) & $0.52 \times 10^{9} / \mathrm{L}$ & $0.55 \times 10^{9} / \mathrm{L}$ \\
\hline Aspirin sensitivity & $77 \%$ & $63 \%$ \\
\hline Asthma & $100 \%$ & $100 \%$ \\
\hline Aeroallergen sensitisation & $100 \%$ & $42 \%$ \\
\hline
\end{tabular}

\section{Discussion}

Our real-life data demonstrates that Omalizumab is an effective intervention for CRSwNP and as far as we are aware we are the first to show that sinonasal improvement is parallel to the improved asthma control at each time point. The improvement seen with Omalizumab approached the treatment efficacy as assessed by the SNOT-22 score recorded after surgery. Such findings clearly support the concept of the one airway hypothesis and highlight a definite role for overlapping IgE driven pathways in both CRSwNP and allergic asthma.

Current asthma treatment guidelines for Omalizumab dictate that only patients with severe treatment refractory allergic asthma with sensitisation to a perennial allergen within a limited total serum IgE range can be treated. Currently, the majority of CRSwNP and associated late onset severe asthma patients do not have skin prick or serum IgE positivity to aeroallergens ${ }^{(1)}$. Given that local tissue IgE driven pathways are active in CRSwNP(5) and non-allergic asthma ${ }^{(6)}$, it is expected that Omalizumab will treat both CRSwNP and asthma in the major 'non-allergic' phenotype. Early data supports this premise in 'non-allergic' $\mathrm{CRSwNP}^{(7)}$ and asthma treated with Omalizumab ${ }^{(8)}$. A growing body of evidence has shown that the most obvious and thus accepted bio-marker in use - total serum lgE, is not the only predictor of treatment response with Omalizumab in allergic asthma and may even be less effective compared to markers of overall T2 inflammation such as FeNO and blood eosinophils ${ }^{(9)}$. This is bourne out by the fact that CRSwNP patients demonstrate striking $\mathrm{T} 2$ inflammation and blood eosinophilia.

The observational study by Vennera et al. ${ }^{(10)}$ reported a decrease in polyp size with less progression to repeat surgery and use of intranasal steroids in an Omalizumab treated cohort of CRSWNP with severe asthma. Using the validated disease assessment tool SNOT-22 and ACQ-7 we are the first to report improvement in both CRSWNP and severe allergic asthma together at time points as early as 4 weeks post treatment (i.e. after just one dose of Omalizumab) and show sustained improvement with continued therapy. These findings are relevant as Omalizumab trials in 


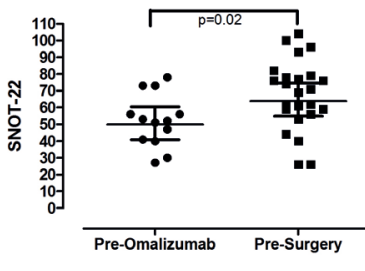

$1 \mathrm{~A}$

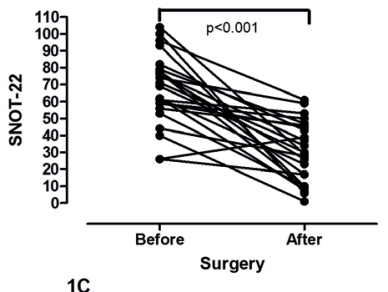

$1 C$

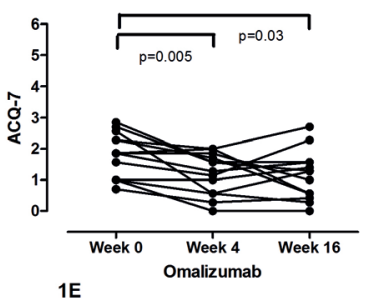

Figure 1. Summary of treatment outcomes.

severe asthma have so far failed to assess nasal outcomes and there are limited studies looking at sinonasal disease in a double blind placebo controlled (DBPC) manner. Such studies are now urgent. Pinto et al. ${ }^{(11)}$ demonstrated in a DBPC trial carried out in a small sample of CRSWNP patients an improvement in SNOT-20 scores over a 6 month period in the Omalizumab arm but other measures of disease response such as olfaction, airflow with NIPF, endoscopic scores and imaging did not show significant improvement. It must be remembered that our Omalizumab group were selected out from asevere refractory asthma phenotype group. An explanation for the lower baseline SNOT-22 score observed in our Omalizumab group may be due to the frequent courses of oral steroids required to stabilise their asthma prior to commencing Omalizumab. It can be speculated that systemic steroids may have modulated immune dysregulation and total IgE levels, which may enhance the efficacy of Omalizumab. The Pinto study patients were selected solely on the basis of CRS. Any conflict in findings from current studies or reports highlight the importance of understanding how to select patients for biologic studies, as we have learned from asthma studies ${ }^{(12)}$. Currently, Omalizumab is a long term treatment for asthma, and is only stopped should the drug stop working or if the patient develops adverse events with drug. Clearly, until further detailed studies are available, given the cost of Omalizumab, one can debate on whether treatment outcomes will be cost-effective in the long run ${ }^{(13)}$. If studies can robustly show that for specified endo-phenotypes where patients with both severe CRSwNP and asthma are effectively treated with Omalizumab, then this approach will be beneficial both in terms of only requiring one drug to treat two severe diseases and in terms of health economics and utilisation of resources by government bodies.

The overall annual economic burden of CRS in the United States is estimated as $\$ 22$ billion (direct and indirect costs) $)^{(14)}$. Repeated surgery for recurrence of disease is common. In between surgery, patients continue to report active disease and poor quality of life. Given the reduction of SNOT scores reported here in patients with severe asthma and CRSwNP, it is possible that this treatment would reduce the requirement for surgical treatment of CRSWNP in these patients. This would be a large cost saving and warrants further study.

Early data confirms the potential for anti-eosinophil strategies with Mepolizumab to target CRSwNP(15). It is too early to speculate if the use of Mepolizumab in asthma will demonstrate benefit in CRSwNP as only a $100 \mathrm{mg}$ subcutaneous dose is licenced for asthma, and the two reported studies for CRSwNP used $750 \mathrm{mg}$ intravenously(15,16). IL-4/1L-13 inhibition with Dupilumab has been shown as clinically effective ${ }^{(17)}$.

Asthma treatment guidelines so far do not consider upper and lower airway disease within a patient as associated conditions, and overall fail to promote the urgent need to address the whole airway together. We appreciate the limitations of our study, and more robust double blind placebo controlled studies are urgently needed to determine which patients will benefit from a 'one airway-one treatment approach'. Such studies must evaluate patient response in broader bio-marker positive subgroups, such that potentially effective biologics are not denied to large cohorts of severe airway patients, as currently is the case. ENT surgeons and respiratory physicians need to treat such patients in a combined approach.

\section{Conclusion}

In conclusion we demonstrate rapid improvement in both CRSwNP and associated severe allergic asthma early on with Omalizumab treatment. The potential to treat the one airway with one drug is on the horizon, but large studies using broad biomarker groups assessing the upper and lower outcomes together are urgently needed. Only then can the right patient be matched with the right biological intervention.

\section{Authorship contribution}

TB, JS, CR and HHK collected and analysed the data. VJL, DSR and HHK supervised data collection, undertook data interpretation. All authors contributed to writing of the manuscript. 


\section{Conflict of interest}

Douglas Robinson and Harsha Kariyawasam have received payment from Novartis for advisory board work.

\section{References}

1. Fokkens WJ, Lund VJ, Mullol J et al. European Position Paper On Rhinosinusitis and Nasal Polyps 2012. Rhinol Suppl 2012 Mar ;( 23):3-298.

2. Lin DC, Chandra RK, Tan BK, Zirkle W, Conley DB, Grammer LC et al. Association between severity of asthma and degree of chronic rhinosinusitis. Am J Rhinol Allergy 2011 Jul; 25(4):205-8.

3. Chung KF, Wenzel SE Brozek JL et al. International ERS/ATS guidelines on definition, evaluation and treatment of severe asthma Eur Respir J 2014; 43: 343-373

4. Toma S, Hopkins C. Stratification of SNOT-22 scores into mild, moderate or severe and relationship with other subjective instruments. Rhinology. 2016 Jun; 54(2):129-33

5. Zhang N, Holtappels G, Gevaert P et al. Mucosal tissue polyclonal lgE is functional in response to allergen and SEB. Allergy 2011. Jan; 66(1):141-8.

6. Lommatzsch M, Korn S, Buhl R, Virchow JC. Against all odds: anti-IgE for intrinsic asthma? Thorax. 2014 Jan; 69(1):94-6

7. Gevaert P, Calus L, Van Zele $T$ et al. Omalizumab is effective in allergicand nonallergic patients with nasal polyps and asthma. J Allergy Clin Immunol. 2013. Jan; 131(1):110-6.

8. Pillai P, Chan YC, Wu SY et al. Omalizumab reduces bronchial mucosal IgE and improves lung function in non-atopic asthma. Eur Respir J. 2016. Dec;48(6):1593-1601

9. Hanania NA, Wenzel S, Rosén $K$ et al. Exploring the effects of omalizumab in allergic asthma: an analysis of biomarkers in the EXTRA study. Am J Respir Crit Care Med. 2013 Apr; 187(8):804-11

10. Vennera Mdel C, Picado C, Mullol J et al. Efficacy of omalizumab in the treatment of nasal polyps. Thorax. 2011. Sep; 66(9):824-5.

11. Pinto JM, Mehta $N$, DiTineo $M$ et al. A randomized, double-blind, placebo-controlled trial of anti-lgE for chronic rhinosinusitis. Rhinology. 2010. Sep; 48(3):318-24

12. Robinson DS, Kariyawasam HH, Heaney LG. Phase three studies of biologics for severe asthma: could do better? Eur Respir J. 2017 Sep; 50(3).

13. Naclerio RM, Baroody FM, Pinto JM. Should clinicians use omalizumab for the treatment of nasal polyps? J Allergy Clin Immunol. 2013. Jul; 132(1):247.

14. Smith KA, Orlandi RR, Rudmik L Cost of adult chronic rhinosinusitis: A systematic review. Laryngoscope. 2015 Jul; 125(7):1547-56

15. Gevaert P, Van Bruaene N, Cattaert T et al. Mepolizumab, a humanized anti-IL-5 mAb, as a treatment option for severe nasal polyposis. J Allergy Clin Immunol 2011 Nov; 128(5):989-95

16. Bachert C, Sousa AR, Lund VJ et al. Reduced need for surgery in severe nasal polyposis with mepolizumab: a randomized trial. J Allergy Clin Immunol. 2017 Jul. 6749(17): 31081-3.

17. Bachert C, Mannent L, Naclerio RM et al. Effect of subcutaneous dupilumab on nasal polyp burden in patients with chronic sinusitis and nasal polyposis: a randomized clinical trial. JAMA. 2016 Feb; 315(5):469-79.

\section{Dr Harsha Kariyawasam PhD FRCP}

Adult Specialist Allergy and Clinica

Immunology

Rhinology Section

Royal National Throat Nose Ear

Hospital and UCLH

330 Grays Inn Road

London WC1X 8DA

United Kingdom

Telephone +44 2034565242

Fax +442034565183

E-mail:

harsha.kariyawasam@nhs.net 